\title{
Le Professeur Alfred MoQuin-Tandon
} (1804-1863)

\author{
Par René-M. NICOLI et Michel QUILICI
}

Il y a cent ans exactement, le 15 avril 1863 , mourait brutalement et prématurément le professeur Alfred Moquin-Tandon, titulaire de la chaire d'Histoire naturelle médicale de la Faculté de Médecine de Paris.

Nous désirerions évoquer brièvement la vie et les idées de cet homme qui eut, sur les Sciences naturelles en général et sur la parasitologie en particulier, une influence considérable et souvent méconnue.

\section{I. - La vie d'Alfred Moquin-Tandon}

Christian-Horace-Bénédict-Alfred Moquin-Tandon naquit à Montpellier le 7 mai 1804. Si son père, Horace Moquin, est pour nous à peu près inconnu - nous savons seulement qu'il occupait une situation aisée dans le commerce et que, de ce fait, il put aider le jeune Alfred à ses débuts —, sa mère, Cécile Tandon, était la petite-fille d'un banquier, André-Auguste Tandon (1759-1824), célèbre par ailleurs comme poète de langue d'oc. La famille du naturaliste était protestante: longtemps exilée à Genève après la révocation de l'Edit de Nantes, elle était revenue, après le décret d'amnistie, se fixer dans la région de Montpellier.

Après de solides études classiques que nul — de nos jours - ne peut effectuer (Alfred Moquin-Tandon avait une connaissance profonde de la langue latine), le père plaça le fils comme employé dans sa propre entreprise commerciale. Cela ne plaisait guère au jeune Alfred qui préférait les occupations intellectuelles : la poésie française ou surtout romane et les sciences naturelles.

L'Université de Montpellier le forma : le 9 décembre 1826 , à 22 ans, il était reçu enfin docteur ès Sciences naturelles en soutenant deux thèses, l'une de botanique sur la tératologie végétale, l'autre de zoologie sur les Hirudinées. Cette dernière fut — paraît-il - fort mal accueillie et le jeune Moquin traité « d'esprit absurde et de gamin »! (Journal de 1834, 58).

Deux ans plus tard, le 18 août 1828, Alfred Moquin devenait docteur en médecine avec une thèse sur la tuberculose (on disait alors phtisie) laryngée. 
Sur les conseils de ses maîtres et tout particulièrement d'Auguste de SaintHilaire (1), le jeune Moquin-Tandon se tourne alors vers l'enseignement.

En 1829-1830, il professe à l'Athénée de Marseille un cours de physiologie comparée. En 1833, il reçoit la charge de la chaire d'Histoire naturelle de la Faculté des Sciences de Toulouse, où il cumule l'enseignement de la géologie, de la zoologie et de la botanique. En février 1834, la ville de Toulouse le nomme professeur au Jardin des Plantes de cette ville et directeur de ce Jardin. C'est une charge écrasante. MoquinTandon décide alors d'aller plaider sa cause au Ministère. C'est son premier voyage à Paris (septembre-octobre 1834). Le ministre Guizot et le baron Thénard lui font d'ailleurs bon accueil et la chaire d'Histoire naturelle se trouva dédoublée: zoologie et botanique d'une part, cours confiés à Moquin-Tandon, géologie d'autre part.

C'est la période calme de la vie de Moquin-Tandon. Vers 1837, il épouse Mlle de Terson de Palleville, originaire de Revel (Haute-Garonne). Trois enfants, deux fils et une fille, naissent de cette union.

En juin 1841, il est reçu l'un des quarante de l'Académie des Jeux floraux, malgré ses convictions protestantes. En son discours d'entrée, il souligne « les alliances de l'Histoire naturelle et de la Littérature ».

Moquin est alors un érudit : il révise, en collaboration avec Ad. Gatien-Arnoult, les « Leys d'Amors du $\mathrm{XIV}^{\circ}$ siècle » et publie de délicieux recueils poétiques : « Margaritedas 》 et « Guindoutelas » (2).

Le 12 mai 1851, Moquin-Tandon devenait membre correspondant de l'Institut.

En août 1852, il fut envoyé en mission en Corse par le Gouvernement afin d'achever les travaux botaniques de l'Avignonnais Esprit Requien, mort l'année précédente. Ce fut alors que Moquin-Tandon fit la connaissance d'un jeune professeur de physique au lycée d'Ajaccio, visite dont l'importance devait être considérable : ce jeune professeur abandonna les mathématiques et la physique pour se pencher sur les coquilles et les insectes : c'était Jean-Henri Fabre...

En 1853, le ministre de l'Instruction publique, H. Fortoul, propose à MoquinTandon la chaire d'Histoire naturelle médicale de la Faculté de Médecine de Paris et la direction du Jardin des Plantes de la Faculté, en remplacement d'Achille Richard, décédé le 5 octobre 1852. Moquin est nommé par décret le 30 avril 1853 . On le préfère à Ch. Martins, agrégé de la Faculté de Médecine de Paris, qui d'ailleurs fut alors nommé professeur d'Histoire naturelle médicale à la Faculté de Médecine de Montpellier. Les étudiants de Moquin-Tandon furent séduits par leur nouveau professeur et s'inclinèrent devant la précision de ses cours, sa vivacité d'esprit et la justesse des dessins que Moquin traçait au tableau noir.

Le 20 février 1854, Moquin-Tandon fut élu membre titulaire à l'Académie des Sciences, en remplacement d'Auguste de Saint-Hilaire, récemment décédé. Il se pré-

(1) Augustin Prouvençal de Saint-Hilaire, botaniste montpélliérain, membre de l'Académie des Sciences (1830) (1799-1853).

(2) Le noyer de Maguelonne (Carya Magalonensis), Toulouse, 1836, est une supercherie littéraire que n'aurait pas désavouée Prosper Mérimée. Moquin publie, en effet, sans nom d'auteur, un de ses poèmes en vieux roman comme issu d'un manuscrit du XIV ${ }^{e}$ siècle. Les spécialistes de l'époque s'y laissèrent prendre ! 
senta contre Louis Pasteur, connu alors comme chimiste, et la lutte fut sévère. En 1857, Moquin fut reçu à l'Académie de Médecine.

A cette époque, Moquin-Tandon, grand fumeur, assez corpulent, présente déjà les premiers signes de l'affection qui va l'emporter. Le 10 avril 1863 , il cède ses cours à un assistant, le docteur Henri-Ernest Baillon. Le 15 avril, il meurt subitement, après une crise douloureuse de trois heures, d'un infarctus du myocarde très vraisemblablement.

L'un de ses fils recueillit alors un manuscrit inédit de son père et le publia, sous le pseudonyme de A. Frédol : «Le Monde de la Mer * (Hachette, éditeur), livre de vulgarisation certes, mais aussi magnifique témoignage de l'amour que le disparu portait à la Création...

La chaire d'Histoire naturelle médicale de la Faculté de Médecine de Paris devenait donc vacante. Elle fut confiée le 22 novembre 1863 à Henri Baillon.

\section{II. - Les règles morales et les idées d'Alfred Moquin-Tandon}

Pour ceux qui le côtoyaient, le professeur Moquin-Tandon était, peut-être plus encore qu'un savant, un être d'élite forçant toutes les sympathies.

Plein de bonhomie, même malicieux, mais également rigide, « ennemi des privilèges, ennemi du favoritisme, méprisant l'adulation, ne demandant rien qu'au travail » (3), Moquin-Tandon était aussi parfois d'un abord froid et même décevant (4). C'est qu'il y avait en lui un être conscient sans doute de sa valeur, mais aussi de ses limites, ce qui parfois devait l'intimider. Il n'en rougissait d'ailleurs pas et avouait sans peine son excessive sensibilité (5).

L'activité scientifique du naturaliste se déroula sur plusieurs plans, car MoquinTandon était à la fois, ce qui est si exceptionnel aujourd'hui, un botaniste, un zoologiste, un médecin et un philosophe.

En Botanique, élève de Prouvençal de Saint-Hilaire - son maître vénéré - et au-delà de Michel Dunal et d'Auguste-Pyrame de Candolle, Moquin-Tandon s'est consa. cré à plusieurs problèmes différents ;

a) l'étude monographique d'une famille végétale, les Chénopodiacées, famille peu brillante (6), mais pleine d'intérêt en raison de ses nombreuses espèces et de leurs habitats si variés ;

b) l'étude biologique des phénomènes térato'ogiques (dédoublements et fasciations) ;

c) la floristique. Notons toutefois que, malgré sa mission en Corse, Moquin-Tandon ne fit rien paraître personnellement sur ce sujet (7).

(3) R. Blanchard, Archives de Parasitologie ; XI (1907), 481.

(4) Voir les paroles de J.-H. Fabre reprises par le biographe de celui-ci, le docteur Gros (Entretien de Sérignan).

(5) La vue du sang d'une égratignure lui était pénible! (cité par le docteur Gros).

(6) Moquin les nommait plaisamment «Les Crapauds du règne végétal ».

(7) Les résultats des travaux de Requien furent publiés en 1852 à Ajaccio (Catalogue des végétaux ligneux qui croissent naturellement en Corse ou qui y sont généralement cultivés. G. Marchi, imprimeur-libraire). Ce travail est presque inconnu. Il est possible qu'Alfred Moquin-Tandon ait contribué à sa parution. Le manuscrit toutefois a été signé à Ajaccio le 3 février 1849 par Requien seul. Une réimpression a été donnée en Avignon en 1868 (Imprimerie de Fr. Seguin). (J. Briquet, Prodrome de la Flore Corse, 1910, p. L). 
En Zoologie, Moquin-Tandon se pencha sur deux groupes d'Invertébrés :

a) les Hirudinées. Il fut le fondateur de l'hirudiniculture et, grâce à sa théorie des zoonites, jeta un jour nouveau sur la biologie de ces Annélides ;

b) les Mollusques terrestres et fluviatiles de France, vus non seulement dans leur systématique, mais encore dans leur anatomie et leur comportement.

De la Conchyliologie, Moquin devait passer à l'Océanographie, avec tout l'enthousiasme d'un poète. La mort seule interrompit cette dernière orientation de la vie du naturaliste (8).

Sa formation était celle d'un naturaliste. Pourtant, son enseignement à la Faculté de Médecine de Paris fit de iui un médecin. Moquin-Tandon souligne \& l'esprit professionnel qui doit dominer toutes les études dans une Facu'té de Médecine ou dans une Eco'e de Pharmacie » (9). Certes, il note le caractère «plus pratique que véritablement scientifique » de ces études et peut-être le déplore un peu, mais en revanche en voit toute la commodité.

C'était - et il y a là quelque chose de très nouveau dans la conception de l'Histoire naturelle médicale - toute une orientation nouvelle.

Il suffit de parcourir le plan des Eléments de Zoo'ogie médicale pour saisir pleinement ce fait : 23 pages sont réservées à l'Anthropo'ogie, 24 à la Systématique, 135 aux * Animaux employés en Médecine * et 210 aux animaux nuisibles, venimeux et parasites. Moquin-Tandon avait parfaitement saisi que l'Histoire naturelle médicale présentait un dénominateur commun, l'Homme, mais qu'en réalité elle gravitait entre deux pôles sans points communs: la Matière médicale et la Parasitologie.

Plus tard, du vivant d'Ernest Baillon, entre 1883 et 1892, Raphaël Blanchard, alors agrégé, reprendra ce principe et orientera la Zoo'ogie médicale définitivement vers la Parasitologie (10).

Il reste bien des aspects méconnus dans l'œuvre d'Alfred Moquin-Tandon qui font de lui un précurseur assez original dans le monde scientifique du milieu du $\mathrm{xIX}^{\circ}$ siècle.

Il fut un systématicien convaincu et même prophétique.

A la fin de sa vie, en effet, la Systématique générale est encore la Systématique cuviérienne, à peine modifiée par des natura!istes comme $\mathrm{H}$. de Blainville et V. Carus. Les grandes divisions du règne animal n'apparaissent pas encore bien clairement. Moquin-Tandon, frappé de l'impossibiiité d'une classification linéaire, propose une classification d'un type nouveau sous la forme d'un plan. Il conçoit ainsi une division des animaux en trois sous-règnes: les Isolés, les Zoonites, les Agrégés, en insistant sur les rapports d'association pouvant exister entre les individus.

Ainsi se distribuent six embranchements :

(8) Le monde de la Mer eut d'ailleurs une grosse influence sur l'orientation des recherches océanographiques.

(9) Eléments de Zoologie médicale, préface I.

(10) Ernest Baillon, décédé le 18 juillet 1895 , ne fut pas immédiatement remplacé, en raison de la réorganisation des études médicales. Sa chaire ne fut pourvue que le 25 juillet 1897 et attribuée à R. Blanchard. Celui-ci obtint en novembre 1906 la transformation de cette chaire d'Histoire naturelle médicale en chaire de Parasitologie et Histoire naturelle médicale. 
Isolés

Zoonites

Agrégés

1. Ostéozoaires (Vertébrés).

2. Malacozoaires (Mollusques).

3. Protozoaires (Hétéromorphes).

4. Entomozoaires (Annelés).

5. Actinozoaires (Radiés).
6. Phytozoaires

(Zoophytes).

Certes, nous savons aujourd'hui combien cette distribution est artificielle, mais Moquin-Tandon insiste déjà sur la nature du système nerveux : "Les animaux à système nerveux ganglionnaire sont rapprochés dans le sens horizontal. 》 (Eléments de Zoologie, 43-47).

Et, pourtant, Moquin-Tandon - grand ami cependant des Geoffroy Saint-Hilaire, Isidore surtout - ne parle pas souvent des théories de i'Evolution. Reste-t-il neutre ? Certainement pas, et certains mots de Moquin-Tandon évoquent l'évo.utionnisme theilardien moderne : «L'Homme est le terme supérieur et définitif de la Création. » (Eléments de Zoo'ogie, 23). Que la vie soit une succession de création ou le fruit d'une évolution progressive, Moquin sagement n'en décide point, mais ii souligne cependant cette ascension de la vie vers l'Homme : «l'Homme, le premier des êtres animés ».

\section{Principaux ouvrages d'Alfred Moquin-Tandon}

1826. - Monographie des Hirudinées. Thèse doctorat ès Sciences (Zoologie), Faculté de Montpellier, in- $4^{\circ}, 152$ p., 7 pl. col. (édité en 1827).

1826. - Essai sur les dédoublements ou multiplication d'organes dans les végétaux. Thèse doctorat ès Sciences (Botanique), Faculté de Montpellier, Paris et Montpellier, Gabon, in- $4^{\circ}$.

1828. - La phtisie laryngée. Thèse doctorat en Médecine, Faculté de Montpellier.

1840. - Chenopodearum monographica enumeratio auctore A. Moquin-Tandon, Paris, J. Loos, in- $8^{\circ}, \mathrm{xI}+182 \mathrm{p}$.

1841. - Eléments de tératologie végétale ou Histoire des anomalies dans l'organisation des végétaux, Paris, P. J. Loos, in- $8^{\circ}, \mathrm{XIII}+403 \mathrm{p}$.

1846. - Monographie de la famille des Hirudinées. Nouvelle édition revue et augmentée, Paris, Baillière, in- $8^{\circ}, 450$ p., atlas, 14 pl. col.

1849. - Prodromus systematis naturalis regnis vegetabilis d'A.P. de Candolle, vol. XIII. $2^{\mathrm{e}}$ partie: Monochlamydées. Paris, Masson, $458 \mathrm{p}$.

1855. - Histoire naturelle des Mollusques terrestres et fluviatiles de France contenant des études générales sur leur anatomie et leur physiologie et la description particulière des genres, des espèces et des variétés. Paris, Baillière, 2 vol. in- $8^{\circ}, 450$ p., atlas, 54 pl. n. ou col.

1860. - Eléments de Zoologie médicale contenant la description détaillée des animaux utiles à la Médecine et des Espèces nuisibles à l'Homme, particulièrement des venimeuses 
et des parasites, précédés de considérations générales sur lorganisation et sur la classification des animaux et d'un résumé sur l'Histoire naturelle de l'Homme. Paris, Baillière, in-18, xvI +428 p., 122 fig.

1861. - Eléments de Botanique médicale contenant la description des végétaux utiles à la Médecine et des Espèces nuisibles à l'Homme, vénéneuses ou parasites précédés de considérations sur l'organisation et la classification des végétaux. Paris, Baillière, in-18, $\mathrm{xx}+543$ p., 128 fig.

1865. - Le Monde de la Mer (A. Frédol). Paris, Hachette, 632 p., 200 fig., 21 pl. col., $2^{\circ}$ édition : 1866.

1893. - Lettres à Auguste de Saint-Hilaire, 1837-1851.

1944. - Journal de voyage inédit (1834) publié par M. Roland sous le titre: Un naturaliste à Paris sous Louis-Philippe. Paris, Mercure de France, $1^{\text {re }}$ édition : $1944 ; 2^{\circ}$ édition : 1944.

(Laboratoire de Parasitologie, Faculté de Médecine, Marseille) 